\title{
On the liouville intergrability of Lotka-Volterra systems
}

\author{
Pantelis A. Damianou ${ }^{1 *}$ and Fani Petalidou ${ }^{2}$ \\ 1 Department of Mathematics and Statistics, University of Cyprus, Nicosia, Cyprus \\ ${ }^{2}$ Department of Mathematics, Aristotle University of Thessaloniki, Thessaloniki, Greece
}

\section{Edited by:}

Oleg N. Kirillov, Helmholtz-Zentrum

Dresden-Rossendorf, Germany

\section{Reviewed by:}

Petre Birtea, West University of

Timisoara, Romania

Mark Levi, Pennsy/vania State

University, USA

Igor Mencattini, Universidade de

São Paulo, Brazil

\section{*Correspondence:}

Pantelis A. Damianou, Department

of Mathematics and Statistics,

University of Cyprus, PO Box 20537,

Nicosia 1678, Cyprus

e-mail:damianou@ucy.ac.cy
This paper is a review on some recent works on the Liouville integrability of a large class of Lotka-Volterra systems.

Keywords: Lotka-Volterra equations, integrable systems, poisson geometry, integrable models, hamiltonian systems.

\section{INTRODUCTION}

The Lotka-Volterra equations were discovered independently by Alfred Lotka and Vito Volterra around 1925. Volterra was trying to make sense of the fact that the predator fish increased in numbers after WWI. This question was posed to him by his sonin-law Umberto D'Ancona a marine biologist who collected data of fish catches in the Adriatic for the years during and after the war. Volterra proposed the following simple system to model the interaction between predator and prey fish

$$
\begin{aligned}
& \dot{x}=x(a-b y) \\
& \dot{y}=y(-c+d x)
\end{aligned}
$$

where $a, b, c, d>0$. This system and its generalizations to $n$ dimensions is one of the most basic models in population dynamics. The variable $x$ denotes the density of prey fish while $y$ is the density of predator fish. Note that if there are no predators $(y=0)$ then $x$ grows at a constant rate $\dot{x}=a x$, the so called Malthusian law of population. Volterra made the assumption that the interaction between predator and prey fish depends on both $x$ and $y$, hence the Malthusian law is modified by subtracting a term $b x y$. Note that he did not take into account a possible death of prey fish due to other causes. Similarly, the density of the predator fish increases at a rate proportional to both $x$ and $y$, i.e., a factor $d x y$. Assuming that they die at the rate $\dot{y}=-c y$ we get the second equation. The same model was also derived by Lotka [1] in the context of chemical reaction theory.

Note that the vector field vanishes at the origin $(0,0)$ and at the point $\left(\frac{c}{d}, \frac{a}{b}\right)$. The origin is saddle point while the second point is a center, i.e., it corresponds to a periodic solution. It is not difficult to produce a constant of motion. We multiply the first equation by $\frac{c-d x}{x}$ and the second by $\frac{a-b y}{y}$ and then we add the result. We obtain

$$
\frac{\dot{x}}{x}(c-d x)+\frac{\dot{y}}{y}(a-b y)=0 .
$$

This equation is equivalent to

$$
\frac{d}{d t}(c \ln x-d x+a \ln y-b y)=0 .
$$

Therefore the function

$$
H(x, y)=c \ln x+a \ln y-d x-b y
$$

is a constant of motion. The function $H$ is actually a Hamiltonian. By defining the Poisson bracket on $\mathbb{R}^{2}$ by $\{x, y\}=x y$ we produce the following Hamiltonian formulation

$$
\begin{aligned}
& \dot{x}=\{x, H\}=x(a-b y) \\
& \dot{y}=\{y, H\}=y(-c+d x) .
\end{aligned}
$$

The Lotka-Volterra equations generalize from two to $n$ species. The most general form of the equations is

$$
\dot{x}_{i}=\varepsilon_{i} x_{i}+\sum_{j=1}^{n} a_{i j} x_{i} x_{j}, \quad i=1,2, \ldots, n,
$$

where $x_{i}$ denotes the density of the $i$ th species, $\varepsilon_{i}$ is its intrinsic growth (or decay) rate and the matrix $A=\left(a_{i j}\right)$ is called the interaction matrix. We consider Lotka-Volterra equations without linear terms $\left(\varepsilon_{i}=0\right)$, i.e., the population of the ith species stays constant if there is no interraction with other species. We also assume that the matrix of interaction coefficients $A=\left(a_{i j}\right)$ is skew-symmetric. This assumption places the problem in the context of the so called conservative Lotka-Volterra systems. 
These systems can be written in Hamiltonian form using the Hamiltonian function

$$
H=x_{1}+x_{2}+\cdots+x_{n} .
$$

Hamilton's equations take the form $\dot{x}_{i}=\left\{x_{i}, H\right\}=\sum_{j=1}^{n} \pi_{i j}$ with quadratic functions

$$
\pi_{i j}=\left\{x_{i}, x_{j}\right\}=a_{i j} x_{i} x_{j}, \quad i, j=1,2, \ldots, n .
$$

From the skew symmetry of the matrix $A=\left(a_{i j}\right)$ it follows that the Schouten-Nijenhuis bracket $[\pi, \pi]$ vanishes identically:

$$
\begin{aligned}
{[\pi, \pi]_{i j k} } & =2\left(a_{i j}\left\{x_{i} x_{j}, x_{k}\right\}+a_{j k}\left\{x_{j} x_{k}, x_{i}\right\}+a_{k i}\left\{x_{k} x_{i}, x_{j}\right\}\right) \\
& =2\left(a_{i j}\left(a_{j k}+a_{i k}\right)+a_{j k}\left(a_{k i}+a_{j i}\right)+a_{k i}\left(a_{i j}+a_{k j}\right)\right) x_{i} x_{j} x_{k} \\
& =0 .
\end{aligned}
$$

The bivector field $\pi$ is an example of a diagonal Poisson structure.

The Poisson tensor (2) is Poisson isomorphic to the constant Poisson structure defined by the constant matrix $A$, see [2]. If $\mathbf{k}=$ $\left(k_{1}, k_{2} \cdots, k_{n}\right)$ is a vector in the kernel of $A$ then the function

$$
f=x_{1}^{k_{1}} x_{2}^{k_{2}} \cdots x_{n}^{k_{n}}
$$

is a Casimir of $\pi$. Indeed for an arbitrary function $g$ the Poisson bracket $\{f, g\}$ is

$$
\{f, g\}=\sum_{i, j=1}^{n}\left\{x_{i}, x_{j}\right\} \frac{\partial f}{\partial x_{i}} \frac{\partial g}{\partial x_{j}}=\sum_{j=1}^{n}\left(\sum_{i=1}^{n} a_{i j} k_{i}\right) x_{j} f \frac{\partial g}{\partial x_{j}}=0 .
$$

If the matrix $A$ has rank $r$ then there are $n-r$ functionally independent Casimirs. This type of integral can be traced back to Volterra [3]; see also [2, 4, 5].

The most famous special case of Lotka-Volterra system is the KM system (also known as the Volterra system) defined by

$$
\dot{x}_{i}=x_{i}\left(x_{i+1}-x_{i-1}\right) \quad i=1,2, \ldots, n,
$$

where $x_{0}=x_{n+1}=0$. It was first solved by Kac and vanMoerbeke [6], using a discrete version of inverse scattering due to Flaschka [7]. In Moser [8] Moser gave a solution of the system using the method of continued fractions, and in the process he constructed action-angle coordinates. Lax pairs of the system can be found in Moser [8], Damianou [9]. Equations (3) can be considered as a finite-dimensional approximation of the Korteweg-de Vries (KdV) equation. This system has a close connection with the Toda lattice,

$$
\begin{array}{ll}
\dot{a}_{i}=a_{i}\left(b_{i+1}-b_{i}\right) & i=1, \ldots, n-1 \\
\dot{b}_{i}=2\left(a_{i}^{2}-a_{i-1}^{2}\right) & i=1, \ldots, n .
\end{array}
$$

In fact, a transformation of Hénon connects the two systems:

$$
\begin{aligned}
& a_{i}=-\frac{1}{2} \sqrt{x_{2 i} x_{2 i-1}} \quad i=1, \ldots, n-1 \\
& b_{i}=\frac{1}{2}\left(x_{2 i-1}+x_{2 i-2}\right) \quad i=1, \ldots, n .
\end{aligned}
$$

The Lotka-Volterra system forms the basis for many models used today in the analysis of population dynamics. It has other applications in Physics, e.g., laser Physics, plasma Physics (as an approximation to the Vlasov-Poisson equation), and neural networks. It appears also in computer science, e.g., communication networks, see [10]. Lotka-Volterra systems have been studied extensively, see e.g., $[4,11-14]$. The Darboux method of finding integrals of finite dimensional vector fields and especially for various types of Lotka-Volterra systems has been used by several authors, e.g., [15-20].

\section{HAMILTONIAN STRUCTURE}

There is a symplectic realization of the Lotka-Volterra system which goes back to Volterra. For simplicity we write the equations in the form

$$
\dot{x}_{j}=\sum_{k=1}^{n} a_{j k} x_{j} x_{k}, \text { for } j=1,2, \ldots, n,
$$

where the matrix $A=\left(a_{j k}\right)$ is a fixed skew-symmetric matrix. In Fernandes and Oliva [21] the Hamiltonian formulation is obtained based on Volterra's work using a symplectic realization from $\mathbf{R}^{2 n} \mapsto \mathbf{R}^{n}$. Volterra defined the variables

$$
q_{i}(t)=\int_{0}^{t} x_{i}(s) d s
$$

(which he called quantity of life) and

$$
p_{i}(t)=\ln \left(\dot{q}_{i}\right)-\frac{1}{2} \sum_{k=1}^{n} a_{i k} q_{k}
$$

for $i=1,2, \ldots, n$. Now, the variables are doubled and the Volterra's transformation is:

$$
\begin{aligned}
\mathbf{R}^{2 n} & \mapsto \mathbf{R}^{n} \\
\left(q_{1}, \ldots, q_{n}, p_{1}, \ldots, p_{i}\right) & \mapsto\left(x_{1}, \ldots, x_{n}\right),
\end{aligned}
$$

where

$$
x_{i}=e^{p_{i}+\frac{1}{2} \sum_{k=1}^{n} a_{i k} q_{k}}, \text { for } i=1,2, \ldots, n \text {. }
$$

The Hamiltonian in these $(q, p)$ coordinates becomes

$$
H=\sum_{i=1}^{n} x_{i}=\sum_{i=1}^{n} \dot{q}_{i}=\sum_{i=1}^{n} e^{p_{i}+\frac{1}{2} \sum_{k=1}^{n} a_{i k} q_{k}} .
$$

The vector field (4) can be written as

$$
\begin{aligned}
& \dot{q}_{i}=\frac{\partial H}{\partial p_{i}}=\left\{q_{i}, H\right\}, \\
& \dot{p}_{i}=-\frac{\partial H}{\partial q_{i}}=\left\{p_{i}, H\right\},
\end{aligned}
$$


for $i=1,2, \ldots, n$, where the bracket $\{\cdot, \cdot\}$ is the standard symplectic on $\mathbb{R}^{2 n}$, that is:

$$
\left\{q_{i}, p_{j}\right\}=\delta_{i j}=\left\{\begin{array}{l}
1, \text { if } i=j \\
0, \text { if } i \neq j
\end{array}, \text { for } i, j=1,2, \ldots, n\right. \text {. }
$$

All others are equal to zero. This system has $n$ integrals given by

$$
I_{j}\left(q_{j}, p_{j}\right)=p_{j}-\frac{1}{2} \sum_{k=1}^{n} a_{j k} q_{k}
$$

One checks that indeed

$$
\dot{I}_{j}=\left\{I_{j}, H\right\}=0 \text {. }
$$

Moreover, $\left\{I_{j}, J_{k}\right\}=a_{j k}$. The corresponding Poisson bracket produced by the transformation (5) in the $x$ coordinates is

$$
\left\{x_{i}, x_{j}\right\}=a_{i j} x_{i} x_{j}, \text { for } i, j=1,2, \ldots, n \text {. }
$$

This observation gives a another proof that the bracket (2) is indeed Poisson.

\section{BOGOYAVLENSKY-VOLTERRA SYSTEMS}

We now describe the construction of the generalized Volterra systems of Bogoyavlensky (see $[22,23]$ ). In this first construction the matrix $A$ is not skew-symmetric but we include the details for completeness.

Let $\mathfrak{g}$ be a simple Lie algebra, with $\operatorname{dim} \mathfrak{g}=n$, and let $\Pi=$ $\left\{\omega_{1}, \omega_{2}, \ldots, \omega_{n}\right\}$ be a Cartan-Weyl basis for the simple roots in $\mathfrak{g}$. There exist unique positive integers $k_{i}$ such that

$$
k_{0} \omega_{0}+k_{1} \omega_{1}+\cdots+k_{n} \omega_{n}=0,
$$

where $k_{0}=1$ and $\omega_{0}$ is the minimal negative root. We consider the Lax pair:

$$
\dot{L}=[B, L],
$$

where

$$
\begin{aligned}
L(t) & =\sum_{i=1}^{n} b_{i}(t) e_{\omega_{i}}+e_{\omega_{0}}+\sum_{1 \leq i<j \leq n}\left[e_{\omega_{i}}, e_{\omega_{j}}\right], \\
B(t) & =\sum_{i=1}^{n} \frac{k_{i}}{b_{i}(t)} e_{-\omega_{i}}+e_{-\omega_{0}} .
\end{aligned}
$$

Let $\mathfrak{h} \subset \mathfrak{g}$ be the Cartan subalgebra. For every root $\omega_{\mathfrak{a}} \in \mathfrak{h}^{*}$ there is a unique $H_{\omega_{a}} \in \mathfrak{h}$ such that $\omega(h)=\beta\left(H_{\omega_{a}}, h\right)$, for all $h \in \mathfrak{h}$, where $\beta$ denotes the Killing form. Also, $\beta$ induces an inner product on $\mathfrak{h}^{*}$ by setting $\left\langle\omega_{a}, \omega_{b}\right\rangle=\beta\left(H_{\omega_{a}}, H_{\omega_{b}}\right)$, and we define

$$
c_{i j}=\left\{\begin{aligned}
& 1 \text { if }\left\langle\omega_{i}, \omega_{j}\right\rangle \neq 0 \text { and } i<j, \\
& 0 \text { if }\left\langle\omega_{i}, \omega_{j}\right\rangle=0 \text { or } i=j, \\
&-1 \text { if }\left\langle\omega_{i}, \omega_{j}\right\rangle \neq 0 \text { and } i>j .
\end{aligned}\right.
$$

With these choices, the Lax pair above is equivalent to the system of o.d.e.'s

$$
\dot{b}_{i}=-\sum_{j=1}^{n} \frac{k_{j} c_{i j}}{b_{j}}
$$

To obtain a Lotka-Volterra type system one introduces a new set of variables by

$$
\begin{aligned}
& x_{i j}=c_{i j} b_{i}^{-1} b_{j}^{-1}, \\
& x_{j i}=-x_{i j}, \\
& x_{j j}=0 .
\end{aligned}
$$

Note that $x_{i j} \neq 0$ iff there exists an edge in the Dynkin diagram for the Lie algebra $\mathfrak{g}$ connecting the vertices $\omega_{i}$ and $\omega_{j}$. System (7), in the variables $x_{i j}$, takes the form

$$
\dot{x}_{i j}=x_{i j} \sum_{s=1}^{n} k_{s}\left(x_{i s}+x_{j s}\right)
$$

which is a Lotka-Volterra type system.

For example the following system is an open version of a $B_{n}$ system:

$$
\begin{aligned}
& \dot{x}_{1}=x_{1} x_{2}, \quad \dot{x}_{2}=x_{2}\left(x_{3}-x_{1}\right), \\
& \dot{x}_{3}=x_{3}\left(x_{4}-x_{2}\right), \dot{x}_{4}=-x_{4}\left(x_{3}+x_{4}\right) .
\end{aligned}
$$

The Hamiltonian formulation of these systems, Lax pairs and master symmetries were considered by Kouzaris [24]. There is also a Lax pair in Damianou and Fernandes [25]. The system in our example has two integrals of motion, one of degree 2 and one of degree 4 . The quadratic integral is

$$
F_{1}=x_{1}^{2}+x_{2}^{2}+x_{3}^{2}+2 x_{1} x_{2}+2 x_{2} x_{3}+2 x_{3} x_{4}
$$

The fourth degree invariant is

$$
\begin{aligned}
F_{2}= & x_{1}^{4}+x_{2}^{4}+x_{3}^{4}+4 x_{1}^{2} x_{2} x_{3}+6 x_{1}^{2} x_{2}^{2}+4 x_{1} x_{2} x_{3} x_{4}+4 x_{3}^{2} x_{4}^{2} \\
& +4 x_{3} x_{4} x_{2}^{2}+4 x_{1} x_{2}^{3}+4 x_{3}^{3} x_{4}+4 x_{1}^{3} x_{2}+8 x_{3}^{2} x_{2} x_{4} \\
& +8 x_{1} x_{3} x_{2}^{2}+4 x_{1} x_{2} x_{3}^{2}+4 x_{2}^{3} x_{3}+4 x_{2} x_{3}^{3}+6 x_{2}^{2} x_{3}^{2} .
\end{aligned}
$$

\section{MORE BOGOYAVLENSKY'S TYPE SYSTEMS}

Bogoyavlensky in $[22,23]$ and [5] has generalized the KM-system in the following way,

$$
\dot{x}_{i}=x_{i}\left(\sum_{j=1}^{p} x_{i+j}-\sum_{j=1}^{p} x_{i-j}\right)
$$

with periodic condition $x_{n+i}=x_{i}$. We will denote this system with $B(n, p)$. All the results in this section, except the bihamiltonian pair follow [5]. The system has a Lax pair of the form

$$
\dot{L}=[L, A] \text {, }
$$


where $L=X+\lambda M$ and $A=b-\lambda M^{p+1}$. The matrix $X$ has the form $x_{i, i-p}=x_{i}$ for $p+1 \leq i \leq n$ and $x_{i, i+n-p}=x_{i}$ for $1 \leq i \leq$ $p$. The matrix $M$ is defined by $m_{i, i+1}=m_{n, 1}=1$. The matrix $b$ is diagonal with entries $b_{i i}=-\left(x_{i}+x_{i+1}+\cdots+x_{i+p}\right)$.

Example 1. Let us consider the system $B(6,2)$, i.e., $n=6, p=2$. The equations of motion become

$$
\begin{aligned}
& \dot{x}_{1}=x_{1}\left(x_{2}+x_{3}-x_{5}-x_{6}\right) \\
& \dot{x}_{2}=x_{2}\left(x_{3}+x_{4}-x_{1}-x_{6}\right) \\
& \dot{x}_{3}=x_{3}\left(x_{4}+x_{5}-x_{2}-x_{1}\right) \\
& \dot{x}_{4}=x_{4}\left(x_{5}+x_{6}-x_{3}-x_{2}\right) \\
& \dot{x}_{5}=x_{5}\left(x_{6}+x_{1}-x_{4}-x_{3}\right) \\
& \dot{x}_{6}=x_{6}\left(x_{1}+x_{2}-x_{5}-x_{4}\right) .
\end{aligned}
$$

We have

$$
\begin{aligned}
X & =\left(\begin{array}{cccccc}
0 & 0 & 0 & 0 & x_{1} & 0 \\
0 & 0 & 0 & 0 & 0 & x_{2} \\
x_{3} & 0 & 0 & 0 & 0 & 0 \\
0 & x_{4} & 0 & 0 & 0 & 0 \\
0 & 0 & x_{5} & 0 & 0 & 0 \\
0 & 0 & 0 & x_{6} & 0 & 0
\end{array}\right), \\
M & =\left(\begin{array}{llllll}
0 & 1 & 0 & 0 & 0 & 0 \\
0 & 0 & 1 & 0 & 0 & 0 \\
0 & 0 & 0 & 1 & 0 & 0 \\
0 & 0 & 0 & 0 & 1 & 0 \\
0 & 0 & 0 & 0 & 0 & 1 \\
1 & 0 & 0 & 0 & 0 & 0
\end{array}\right), \\
L & =\left(\begin{array}{cccccc}
0 & \lambda & 0 & 0 & x_{1} & 0 \\
0 & 0 & \lambda & 0 & 0 & x_{2} \\
x_{3} & 0 & 0 & \lambda & 0 & 0 \\
0 & x_{4} & 0 & 0 & \lambda & 0 \\
0 & 0 & x_{5} & 0 & 0 & \lambda \\
\lambda & 0 & 0 & x_{6} & 0 & 0
\end{array}\right) .
\end{aligned}
$$

Let $p(x)=\operatorname{det}(L-x I)$ be the characteristic polynomial of $L$. Then the coefficient of $x^{3}$ is of the form $H \lambda^{2}+F_{2}$, where $H=x_{1}+$ $x_{2}+x_{3}+x_{4}+x_{5}+x_{6}$ is the Hamiltonian and $F_{2}=x_{1} x_{3} x_{5}+$ $x_{2} x_{4} x_{6}$. On the other hand the constant term of $p(x)$ has the form $F_{3} \lambda^{2}+F_{4}$, where $F_{3}=x_{1} x_{2} x_{4} x_{5}+x_{1} x_{3} x_{4} x_{6}+x_{2} x_{3} x_{5} x_{6}$ and $F_{4}=x_{1} x_{2} x_{3} x_{4} x_{5} x_{6}$.

By examining the eigenvectors of the coefficient matrix of (11) we can see that the functions $C_{1}=x_{2} x_{5}, C_{2}=x_{1} x_{4}, C_{3}=x_{2} x_{4} x_{6}$ and $C_{4}=x_{1} x_{3} x_{5}$ are all Casimirs of the corresponding quadratic Poisson structure. Therefore we have a rank 2 Poisson bracket and the system is clearly integrable. It is easy to see that the functions $F_{2}, F_{3}, F_{4}$ can be expressed as functions of $C_{1}, C_{2}, C_{3}, C_{4}$.

Now restrict this system on the invariant submanifold $x_{5}=x_{6}=0$. We obtain the system

$$
\begin{aligned}
& \dot{x}_{1}=x_{1}\left(x_{2}+x_{3}\right) \\
& \dot{x}_{2}=x_{2}\left(x_{3}+x_{4}-x_{1}\right) \\
& \dot{x}_{3}=x_{3}\left(x_{4}-x_{2}-x_{1}\right)
\end{aligned}
$$

$$
\dot{x}_{4}=x_{4}\left(-x_{3}-x_{2}\right) \text {. }
$$

This system is integrable. It has two Casimirs $F_{1}=x_{1} x_{4}=C_{2}$ and $F_{2}=\frac{x_{1} x_{3}}{x_{2}}=\frac{C_{4}}{C_{1}}$.

Example 2. Similarly, the quadratic Poisson structure $\pi_{2}$ associated to the system $B(5,2)$, i.e.,

$$
\pi_{2}=\left(\begin{array}{ccccc}
0 & x_{1} x_{2} & x_{1} x_{3} & -x_{1} x_{4} & -x_{1} x_{5} \\
-x_{1} x_{2} & 0 & x_{2} x_{3} & x_{2} x_{4} & -x_{2} x_{5} \\
-x_{1} x_{3} & -x_{2} x_{3} & 0 & x_{3} x_{4} & x_{3} x_{5} \\
x_{1} x_{4} & -x_{2} x_{4} & -x_{3} x_{4} & 0 & x_{4} x_{5} \\
x_{1} x_{5} & x_{2} x_{5} & -x_{3} x_{5} & -x_{4} x_{5} & 0
\end{array}\right)
$$

has a single Casimir $x_{1} x_{2} x_{3} x_{4} x_{5}$. The system is Hamiltonian with Hamiltonian function

$$
H=x_{1}+x_{2}+x_{3}+x_{4}+x_{5}
$$

and it has as additional first integral the function

$$
F=x_{1} x_{2} x_{4}+x_{1} x_{3} x_{4}+x_{1} x_{3} x_{5}+x_{2} x_{3} x_{5}+x_{2} x_{4} x_{5}
$$

Define the Poisson tensor $\pi_{0}$ as follows:

$$
\pi_{0}=\left(\begin{array}{ccccc}
0 & 0 & -1 & 1 & 0 \\
0 & 0 & 0 & -1 & 1 \\
1 & 0 & 0 & 0 & -1 \\
-1 & 1 & 0 & 0 & 0 \\
0 & -1 & 1 & 0 & 0
\end{array}\right)
$$

It is easy to check that $\pi_{0}$ is compatible with $\pi_{2}$ and that we have a bihamiltonian pair

$$
\pi_{2} d H=\pi_{0} d F
$$

The function $H$ is the Casimir of bracket $\pi_{0}$.

More generally, if $n=2 p+1$, then we can define a (skewsymmetric) tensor field $\pi_{0}$ with non-zero entries $\pi_{0}[i, i+n-p-$ $1]=-1$ for $1 \leq i \leq p+1$ and $\pi_{0}[i, i+n-p]=1$ for $1 \leq i \leq p$. The associated quadratic Poisson structure $\pi_{2}$ to the system (10) and $\pi_{0}$ are compatible and they form a bihamiltonian pair.

Restricting on the submanifold $x_{5}=0$ we obtain the system

$$
\begin{aligned}
& \dot{x}_{1}=x_{1}\left(x_{2}+x_{3}-x_{4}\right) \\
& \dot{x}_{2}=x_{2}\left(x_{3}+x_{4}-x_{1}\right) \\
& \dot{x}_{3}=x_{3}\left(x_{4}-x_{2}-x_{1}\right) \\
& \dot{x}_{4}=x_{4}\left(x_{1}-x_{3}-x_{2}\right) .
\end{aligned}
$$

This system is integrable with second integral given by $x_{1} x_{4}\left(x_{2}+\right.$ $\left.x_{3}\right)$, i.e., the restriction of $F$ on the submanifold.

Example 3. Restricting the $B(7,2)$ on the submanifold $x_{4}=x_{6}=$ $x_{7}=0$ and renaming $x_{5} \rightarrow x_{4}$ results in the following system 


$$
\begin{aligned}
& \dot{x}_{1}=x_{1}\left(x_{2}+x_{3}\right) \\
& \dot{x}_{2}=x_{2}\left(x_{3}-x_{1}\right) \\
& \dot{x}_{3}=x_{3}\left(x_{4}-x_{2}-x_{1}\right) \\
& \dot{x}_{4}=-x_{4} x_{3} .
\end{aligned}
$$

The additional integral is $F=x_{4}\left(x_{1}+x_{2}\right)$.

Example 4. Restricting the $B(7,3)$ on the submanifold $x_{5}=x_{6}=$ $x_{7}=0$ results in the following system

$$
\begin{aligned}
& \dot{x}_{1}=x_{1}\left(x_{2}+x_{3}+x_{4}\right) \\
& \dot{x}_{2}=x_{2}\left(x_{3}+x_{4}-x_{1}\right) \\
& \dot{x}_{3}=x_{3}\left(x_{4}-x_{2}-x_{1}\right) \\
& \dot{x}_{4}=x_{4}\left(-x_{1}-x_{2}-x_{3}\right) .
\end{aligned}
$$

The Poisson matrix in this example is symplectic. The system is integrable since it has two constants of motion $F_{1}=\frac{\left(x_{1}+x_{2}\right) x_{4}}{x_{3}}$ and $F_{2}=\frac{\left(x_{3}+x_{4}\right) x_{1}}{x_{2}}$. Note that

$$
F_{3}=\frac{\left(x_{1}+x_{2}+x_{3}\right)\left(x_{2}+x_{3}+x_{4}\right)}{x_{2}+x_{3}}
$$

is also a first integral.

\section{GENERALIZED VOLTERRA SYSTEMS}

We recall the following procedure from Damianou [26]. Let $\mathfrak{g}$ be any simple Lie algebra equipped with its Killing form $\langle\cdot \mid \cdot\rangle$. One chooses a Cartan subalgebra $\mathfrak{h}$ of $\mathfrak{g}$ and a basis $\Pi$ of simple roots for the root system $\Delta$ of $\mathfrak{h}$ in $\mathfrak{g}$. The corresponding set of positive roots is denoted by $\Delta^{+}$. To each positive root $\alpha$ one can associate a triple $\left(X_{\alpha}, X_{-\alpha}, H_{\alpha}\right)$ of vectors in $\mathfrak{g}$ which generate a Lie subalgebra isomorphic to $\mathfrak{s l}_{2}(\mathbb{C})$. The set $\left(X_{\alpha}, X_{-\alpha}\right)_{\alpha \in \Delta^{+}} \cup\left(H_{\alpha}\right)_{\alpha \in \Pi}$ is a basis of $\mathfrak{g}$, called a root basis. Let $\Pi=\left\{\alpha_{1}, \ldots, \alpha_{\ell}\right\}$ and let $X_{\alpha_{1}}, \ldots, X_{\alpha_{\ell}}$ be the corresponding root vectors in $\mathfrak{g}$. Define

$$
L=\sum_{\alpha_{i} \in \Pi} a_{i}\left(X_{\alpha_{i}}+X_{-\alpha_{i}}\right)
$$

To find the matrix $B$ we use the following procedure. For each $i, j$ form the vectors $\left[X_{\alpha_{i}}, X_{\alpha_{j}}\right]$. If $\alpha_{i}+\alpha_{j}$ is a root then include a term of the form $a_{i} a_{j}\left[X_{\alpha_{i}}, X_{\alpha_{j}}\right]$ in $B$. We make $B$ skewsymmetric by including the corresponding negative root vectors $a_{i} a_{j}\left[X_{-\alpha_{i}}, X_{-\alpha_{j}}\right]$. Finally, we define the system using the Lax equation $\dot{L}=[L, B]$. For a root system of type $A_{n}$ we obtain the KM system.

If a system is of type $A D E$ we can define the system in the following alternative way. Consider the Dynkin diagram of $\mathfrak{g}$ and define a Lotka-Volterra system by the equations

$$
\dot{x}_{i}=x_{i} \sum_{j=1}^{\ell} m_{i j} x_{j},
$$

where the skew-symmetric matrix $m_{i j}$ for $i<j$ is defined to be $m_{i j}=1$ if vertex $i$ is connected with vertex $j$ and 0 otherwise. For $i>j$ the term $m_{i j}$ is defined by skew-symmetry. Note that if we replace one of the $m_{i j}$ for $i<j$ from +1 to -1 we may end up with an inequivalent system. In our definition, the upper part of the matrix $\left(m_{i j}\right)$ consists only of 0 and 1 . However, it is possible to define for each connected graph $2^{m}$ systems, where $m$ is the number of edges, by assigning the \pm 1 sign to each edge. Of course, some of these systems will be isomorphic. One more observation: there are several inequivalent ways to label a graph and therefore the association between graphs and Lotka-Volterra systems is not always a bijection. The number of distinct labellings of a given unlabeled simple graph $G$ on $n$ vertices is known to be

$$
\frac{n !}{|\operatorname{aut}(G)|} \text {. }
$$

Example 5. ( $D_{4}$ system) By examining the Dynkin diagram of the simple Lie algebra of type $D_{4}$ we obtain the system

$$
\begin{aligned}
& \dot{x}_{1}=x_{1} x_{2}, \quad \dot{x}_{2}=-x_{1} x_{2}+x_{2} x_{3}+x_{2} x_{4}, \\
& \dot{x}_{3}=-x_{2} x_{3}, \dot{x}_{4}=-x_{2} x_{4} .
\end{aligned}
$$

One can obtain the same equations in the following way. Define the matrix $L$ using the root vectors of a Lie algebra of type $D_{4}$

$$
L=\left(\begin{array}{cccccccc}
0 & 1 & 0 & 0 & 0 & 0 & 0 & 0 \\
x_{1} & 0 & 1 & 0 & 0 & 0 & 0 & 0 \\
0 & x_{2} & 0 & 1 & 1 & 0 & 0 & 0 \\
0 & 0 & x_{3} & 0 & 0 & 1 & 0 & 0 \\
0 & 0 & x_{4} & 0 & 0 & -1 & 0 & 0 \\
0 & 0 & 0 & x_{4} & -x_{3} & 0 & -1 & 0 \\
0 & 0 & 0 & 0 & 0 & -x_{2} & 0 & -1 \\
0 & 0 & 0 & 0 & 0 & 0 & -x_{1} & 0
\end{array}\right)
$$

and

$$
B=\left(\begin{array}{cccccccc}
0 & 0 & 0 & 0 & 0 & 0 & 0 & 0 \\
0 & 0 & 0 & 0 & 0 & 0 & 0 & 0 \\
x_{1} x_{2} & 0 & 0 & 0 & 0 & 0 & 0 & 0 \\
0 & x_{2} x_{3} & 0 & 0 & 0 & 0 & 0 & 0 \\
0 & x_{2} x_{4} & 0 & 0 & 0 & 0 & 0 & 0 \\
0 & 0 & 0 & 0 & 0 & 0 & 0 & 0 \\
0 & 0 & 0 & x_{2} x_{4} & -x_{2} x_{3} & 0 & 0 & 0 \\
0 & 0 & 0 & 0 & 0 & -x_{1} x_{2} & 0 & 0
\end{array}\right) .
$$

Then the Lax equation $\dot{L}=[L, B]$ is equivalent to (18). We note that

$$
H_{k}=\frac{1}{k} \operatorname{tr} L^{k}, \quad k=1,2, \ldots
$$

are integrals of motion for the system. In fact

$$
\begin{aligned}
4 H_{2}= & x_{1}+x_{2}+x_{3}+x_{4}, \\
4 H_{4}= & \operatorname{tr} L^{4}=x_{1}^{2}+x_{2}^{2}+x_{3}^{2}+x_{4}^{2}+2 x_{1} x_{2} \\
& +2 x_{2} x_{3}+2 x_{2} x_{4}+2 x_{3} x_{4} .
\end{aligned}
$$


Also, the associated quadratic Poisson structure to the system (18) has two Casimirs $F_{1}=x_{1} x_{4}$ and $F_{2}=x_{1} x_{3}$. It turns out that $\operatorname{det}(L)=\left(F_{1}+F_{2}\right)^{2}$.

We have

$$
H_{2}^{2}-4 H_{4}=8\left(x_{1} x_{3}+x_{1} x_{4}\right)=8\left(F_{1}+F_{2}\right) .
$$

We can find the Casimirs by computing the kernel of the matrix

$$
A=\left(\begin{array}{cccc}
0 & 1 & 0 & 0 \\
-1 & 0 & 1 & 1 \\
0 & -1 & 0 & 0 \\
0 & -1 & 0 & 0
\end{array}\right)
$$

The two eigenvectors with eigenvalue 0 are $(1,0,0,1)$ and $(1,0,1,0)$. We obtain the two Casimirs $F_{1}=x_{1}^{1} x_{2}^{0} x_{3}^{0} x_{4}^{1}=x_{1} x_{4}$ and $F_{2}=x_{1}^{1} x_{2}^{0} x_{3}^{1} x_{4}^{0}=x_{1} x_{3}$.

In Charalambides et al. [27] the algorithm was generalized as follows. Consider a subset $\Phi$ of $\Delta^{+}$such that $\Pi \subset \Phi \subset \Delta^{+}$. The Lax matrix is easy to construct

$$
L=\sum_{\alpha_{i} \in \Phi} a_{i}\left(X_{\alpha_{i}}+X_{-\alpha_{i}}\right) .
$$

Here we use the following enumeration of $\Phi$ which we assume to have $m$ elements. The variables $a_{j}$ correspond to the simple roots $\alpha_{j}$ for $j=1,2, \ldots, \ell$. We assign the variables $a_{j}$ for $j=\ell+1, \ell+$ $2, \ldots, m$ to the remaining roots in $\Phi$. To construct the matrix $B$ we use the following algorithm. Consider the set $\Phi \cup \Phi^{-}$which consists of all the roots in $\Phi$ together with their negatives and let $\Psi=\left\{\alpha+\beta \mid \alpha, \beta \in \Phi \cup \Phi^{-}, \alpha+\beta \in \Delta^{+}\right\}$. Define

$$
B=\sum c_{i j} a_{i} a_{j}\left(X_{\alpha_{i}+\alpha_{j}}-X_{-\alpha_{i}-\alpha_{j}}\right)
$$

where $c_{i j}= \pm 1$ if $\alpha_{i}+\alpha_{j} \in \Psi$ with $\alpha_{i}, \alpha_{j} \in \Phi \cup \Phi^{-}$and 0 otherwise. In all eight cases in $A_{3}$ we are able to make the proper choices of the sign of the $c_{i j}$ so that we can produce a Lax pair. This method produces a Lax pair in all but five out of sixty four cases in $A_{4}$. However, when we allow the $c_{i j}$ to take the complex values $\pm i$ we are able to produce a Lax pair in all 64 cases. By using Maple we were able to check that all these examples in $A_{3}$ and $A_{4}$ are in fact Liouville integrable. We will not attempt to prove the integrability of these systems in general due to the complexity of their definition.

This algorithm for certain subsets $\Phi$ recovers well known integrable systems. For example for $\Phi=\Pi$, the simple roots of the root system $A_{n}$, and $c_{i, i+1}=1$ for $i=1,2, \ldots, n-1$ we obtain the KM system while for $\Phi=\Pi \cup\left\{\alpha_{n+1}\right\}$, the simple roots and the highest root, the choice of the signs $c_{i, i+1}=1$ for $i=$ $1,2, \ldots, n-1$ and $c_{1, n+1}=c_{n, n+1}=-1$ produces the periodic KM system.

Example 6. For the root system of type $A_{3}$ if we take $\Phi=$ $\left\{\alpha_{1}, \alpha_{2}, \alpha_{3}, \alpha_{1}+\alpha_{2}\right\}$ then

$$
\Psi=\left\{\alpha_{1}, \alpha_{2}, \alpha_{1}+\alpha_{2}, \alpha_{2}+\alpha_{3}, \alpha_{1}+\alpha_{2}+\alpha_{3}\right\} .
$$

In this example the variables $a_{i}$ for $i=1,2,3$ correspond to the three simple roots $\alpha_{1}, \alpha_{2}, \alpha_{3}$ and the variable $a_{4}$ to the root $\alpha_{1}+\alpha_{2}$. We obtain the following Lax pair:

$$
L=\left(\begin{array}{cccc}
0 & a_{1} & a_{4} & 0 \\
a_{1} & 0 & a_{2} & 0 \\
a_{4} & a_{2} & 0 & a_{3} \\
0 & 0 & a_{3} & 0
\end{array}\right), B=\left(\begin{array}{cccc}
0 & -a_{4} a_{2} & a_{1} a_{2} & -a_{4} a_{3} \\
a_{4} a_{2} & 0 & -a_{1} a_{4} & a_{2} a_{3} \\
-a_{1} a_{2} & a_{1} a_{4} & 0 & 0 \\
a_{4} a_{3} & -a_{2} a_{3} & 0 & 0
\end{array}\right)
$$

Using the substitution $x_{i}=2 a_{i}^{2}$, the system defined by the Lax equation $\dot{L}=[L, B]$ is transformed to the following Lotka-Volterra system.

$$
\begin{array}{ll}
\dot{x_{1}}=x_{1} x_{2}-x_{1} x_{4}, & \dot{x_{2}}=-x_{2} x_{1}+x_{2} x_{3}+x_{2} x_{4}, \\
\dot{x_{3}}=-x_{3} x_{2}+x_{3} x_{4}, & \dot{x_{4}}=x_{4} x_{1}-x_{4} x_{2}-x_{4} x_{3} .
\end{array}
$$

This system is integrable. There exist two functionally independent Casimir functions $F_{1}=x_{1} x_{3}=\operatorname{det} L$ and $F_{2}=x_{1} x_{2} x_{4}$. The standard quadratic Poisson bracket (2) is defined by the relations $\left\{x_{i}, x_{j}\right\}=r_{i, j} x_{i} x_{j}$ where $r_{1,2}=r_{2,3}=r_{3,4}=r_{2,4}=-r_{1,4}=1$ and $r_{1,3}=0$. One can find the Casimirs by computing the kernel of the skew symmetric matrix $A=\left(r_{i, j}\right)_{1 \leq i, j \leq 4}$. The additional integral is the Hamiltonian $H=x_{1}+x_{2}+x_{3}+x_{4}=\operatorname{tr} L^{2}$.

Example 7. Let $\Phi=\left\{\alpha_{1}, \alpha_{2}, \alpha_{3}, \alpha_{1}+\alpha_{2}, \alpha_{2}+\alpha_{3}\right\}$. Its associated Lax equation $\dot{L}=[B, L]$ with

$$
L=\left(\begin{array}{cccc}
0 & a_{1} & a_{4} & 0 \\
a_{1} & 0 & a_{2} & a_{5} \\
a_{4} & a_{2} & 0 & a_{3} \\
0 & a_{5} & a_{3} & 0
\end{array}\right)
$$

and

$$
B=\left(\begin{array}{cccc}
0 & -a_{4} a_{2} & a_{1} a_{2} & -a_{1} a_{5}-a_{4} a_{3} \\
a_{4} a_{2} & 0 & -a_{1} a_{4}-a_{5} a_{3} & a_{2} a_{3} \\
-a_{1} a_{2} & a_{1} a_{4}+a_{5} a_{3} & 0 & -a_{2} a_{5} \\
a_{1} a_{5}+a_{4} a_{3} & -a_{2} a_{3} & a_{2} a_{5} & 0
\end{array}\right)
$$

is equivalent to the following equations of motion

$$
\begin{aligned}
& \dot{a}_{1}=a_{1} a_{2}^{2}-a_{1} a_{5}^{2}-a_{1} a_{4}^{2}-2 a_{3} a_{4} a_{5}, \\
& \dot{a}_{2}=a_{2} a_{4}^{2}+a_{2} a_{3}^{2}-a_{2} a_{1}^{2}-a_{2} a_{5}^{2}, \\
& \dot{a}_{3}=a_{3} a_{5}^{2}+a_{3} a_{4}^{2}-a_{3} a_{2}^{2}+2 a_{1} a_{4} a_{5}, \\
& \dot{a}_{4}=a_{4} a_{1}^{2}-a_{4} a_{2}^{2}-a_{4} a_{3}^{2}, \\
& \dot{a}_{5}=a_{5} a_{1}^{2}-a_{5} a_{3}^{2}+a_{5} a_{2}^{2} .
\end{aligned}
$$


Note that the system is not Lotka-Volterra. It is Hamiltonian with Hamiltonian function $H=\frac{1}{2}\left(a_{1}^{2}+a_{2}^{2}+a_{3}^{2}+a_{4}^{2}+a_{5}^{2}\right)$. The system has Poisson matrix

$$
\pi=\left(\begin{array}{cccccc}
0 & a_{1} a_{2} & -2 a_{4} a_{5} & -a_{1} a_{4} & -a_{1} a_{5} \\
-a_{1} a_{2} & 0 & a_{2} a_{3} & a_{2} a_{4} & -a_{2} a_{5} \\
2 a_{4} a_{5} & -a_{2} a_{3} & 0 & a_{3} a_{4} & a_{3} a_{5} \\
a_{1} a_{4} & -a_{2} a_{4} & -a_{3} a_{4} & 0 & 0 \\
a_{1} a_{5} & a_{2} a_{5} & -a_{3} a_{5} & 0 & 0
\end{array}\right)
$$

of rank 4 . The determinant $C=\left(a_{1} a_{3}-a_{4} a_{5}\right)^{2}$ of $L$ is the Casimir of the system. The trace of $L^{3}$ gives the additional constant of motion

$$
F=\frac{1}{6} \operatorname{tr}\left(L^{3}\right)=a_{1} a_{2} a_{4}+a_{2} a_{3} a_{5} .
$$

Since the three constants of motion are evidently independent, the system is Liouville integrable.

\section{SUBSETS $\Phi$ GIVING RISE TO LOTKA VOLTERRA SYSTEMS}

In Evripidou [28] Evripidou classified all subsets of the positive roots containing the simple roots which give rise to Lotka Volterra systems via the transformation $x_{i}=2 a_{i}^{2}$. He also explicitly described each system associated with this subsets.

Theorem 1. The only choices for the subset $\Phi$ of $\Delta^{+}$so that the corresponding generalized Volterra systems, under the substitution $x_{i}=2 a_{i}^{2}$, are transformed into Lotka-Volterra systems are the following five.

(1) $\Phi=\Pi$,

(2) $\Phi=\Pi \cup\left\{\alpha_{2}+\alpha_{3}+\cdots+\alpha_{n-1}\right\}$,

(3) $\Phi=\Pi \cup\left\{\alpha_{1}+\alpha_{2}+\cdots+\alpha_{n-1}\right\}$,

(4) $\Phi=\Pi \cup\left\{\alpha_{2}+\alpha_{3}+\cdots+\alpha_{n}\right\}$,

(5) $\Phi=\Pi \cup\left\{\alpha_{1}+\alpha_{2}+\cdots+\alpha_{n}\right\}$.

We outline the proof of this theorem. First one proves the theorem for the special case where $\Phi$ is the subset of the positive roots containing the simple roots and only one extra root. This is done by explicitly writing down the matrix $[B, L]$ and setting equal to zero the coefficients of the root vectors corresponding to roots not appearing in $\Phi$. We end up with a linear system of the signs $c_{i, j}$, which in order to have a solution, the extra root $\alpha_{n+1} \in \Phi$ must be of the form $\alpha_{n+1}=\alpha_{k}+\alpha_{k+1}+\ldots+\alpha_{m}$ with $k \leq 2$ and $m \geq n-1$. Since subsystems of Lotka-Volterra systems are also Lotka-Volterra systems, the proof of theorem 1 is a case by case verification of all of the 16 possible subsets $\Phi$ containing the simple roots and roots in

$$
\left\{\alpha_{k}+\alpha_{k+1}+\ldots+\alpha_{m}: k \leq 2 \text { and } m \geq n-1\right\}
$$

Below we describe the corresponding Lotka-Volterra systems.

Case (1) gives rise to the KM system while case (5) gives rise to the periodic KM system.
Case (2) corresponds to the Lax equation $\dot{L}=[L, B]$ with $L$ matrix

$$
L=\left(\begin{array}{cccccccc}
0 & a_{1} & 0 & \cdots & 0 & 0 & 0 & 0 \\
a_{1} & 0 & a_{2} & 0 & & 0 & a_{n+1} & 0 \\
0 & a_{2} & 0 & a_{3} & \ddots & & 0 & 0 \\
\vdots & 0 & a_{3} & \ddots & \ddots & & & 0 \\
0 & & \ddots & \ddots & 0 & a_{n-2} & 0 & \vdots \\
0 & 0 & & & a_{n-2} & 0 & a_{n-1} & 0 \\
0 & a_{n+1} & 0 & & 0 & a_{n-1} & 0 & a_{n} \\
0 & 0 & 0 & 0 & \cdots & 0 & a_{n} & 0
\end{array}\right) .
$$

The skew symmetric matrix $B$ is defined using the method described in the previous section.

After substituting $x_{i}=2 a_{i}^{2}$ for $i=1, \ldots, n+1$, the Lax pair $L, B$ becomes equivalent to the following equations of motion:

$$
\begin{aligned}
\dot{x}_{1} & =x_{1}\left(x_{2}-x_{n+1}\right), \\
\dot{x}_{2} & =x_{2}\left(x_{3}-x_{1}-x_{n+1}\right), \\
\dot{x}_{i} & =x_{i}\left(x_{i+1}-x_{i-1}\right), \quad i=3,4, \ldots, n-2, n \\
\dot{x}_{n-1} & =x_{n-1}\left(x_{n}-x_{n-2}+x_{n+1}\right), \\
\dot{x}_{n+1} & =x_{n+1}\left(x_{1}+x_{2}-x_{n-1}-x_{n}\right) .
\end{aligned}
$$

It is easily verified that for $n$ even, the rank of the corresponding Poisson matrix is $n$ and the function $f=x_{2} x_{3} \cdots x_{n-1} x_{n+1}$ is the Casimir of the system, while for $n$ odd, the rank of the Poisson matrix is $n-1$ and the functions $f_{1}=x_{1} x_{3} \cdots x_{n}=\sqrt{\operatorname{det} L}$ and $f_{2}=x_{2} x_{3} \cdots x_{n-1} x_{n+1}$ are the Casimirs.

Case (3) corresponds to the Lax pair whose Lax matrix $L$ is given by

$$
L=\sum_{i=1}^{n+1} a_{i}\left(X_{\alpha_{i}}+X_{-\alpha_{i}}\right)
$$

with $a_{n+1}=\alpha_{1}+\ldots+\alpha_{n-1}$. The upper triangular part of the skewsymmetric matrix $B$ is

$$
\begin{aligned}
& \sum_{i=1}^{n-1} a_{i} a_{i+1} X_{\alpha_{i}+\alpha_{i+1}}-a_{n-1} a_{n+1} X_{\alpha_{n+1}-\alpha_{n-1}} \\
& -a_{1} a_{n+1} X_{\alpha_{n+1}-\alpha_{1}}-a_{n} a_{n+1} X_{\alpha_{n+1}+\alpha_{n}} .
\end{aligned}
$$

After substituting $x_{i}=2 a_{i}^{2}$ for $i=1, \ldots, n+1$, we obtain the following equivalent equations of motion:

$$
\begin{aligned}
\dot{x}_{1} & =x_{1}\left(x_{2}-x_{n+1}\right) \\
\dot{x}_{i} & =x_{i}\left(x_{i+1}-x_{i-1}\right), i=2,3,4, \ldots, n-2, n \\
\dot{x}_{n-1} & =x_{n-1}\left(x_{n}-x_{n-2}+x_{n+1}\right) \\
\dot{x}_{n+1} & =x_{n+1}\left(x_{1}-x_{n}-x_{n-1}\right) .
\end{aligned}
$$

For $n$ even, the rank of the Poisson matrix is $n$ and the function $f=x_{1} x_{2} \cdots x_{n-1} x_{n+1}$ is the Casimir, while for $n$ odd, the rank of 
the Poisson matrix is $n-1$ and the functions $f_{1}=x_{1} x_{3} x_{5} \cdots x_{n}=$ $\sqrt{\operatorname{det} L}$ and $f_{2}=x_{1} x_{2} \cdots x_{n-1} x_{n+1}$ are Casimirs.

The system obtained in case (4) turns out to be isomorphic to the one in case (3). In fact, the change of variables $u_{n+1-i}=-x_{i}$ for $i=1,2, \ldots, n$ and $u_{n+1}=-x_{n+1}$ in case (3) gives the corresponding system of case (4).

\section{POISSON BRACKETS WITH PRESCRIBED CASIMIRS}

In Damianou and Petalidou [29] by constructing the Poisson brackets for the periodic Toda starting from the well-known Casimirs we observed the surprising appearance of the Volterra system. We first review the basic construction in Damianou and Petalidou [29].

Suppose $\operatorname{dim} M=2 n$. Let $f_{1}, \ldots, f_{2 n-2 k}$ be smooth functions on $M$, functionally independent on a dense open set. Let $\omega_{0}$ be an almost symplectic form on $M$ such that the associate bivector field $\Lambda_{0}$ satisfies:

$$
\begin{aligned}
f & =\left\langle d f_{1} \wedge \ldots \wedge d f_{2 n-2 k}, \frac{\Lambda_{0}^{n-k}}{(n-k) !}\right\rangle \\
& =\left\langle\frac{\omega_{0}^{n-k}}{(n-k) !}, X_{f_{1}} \wedge \ldots \wedge X_{f_{2 n-2 k}}\right\rangle \neq 0,
\end{aligned}
$$

where $X_{f_{i}}=\Lambda_{0}^{\#}\left(d f_{i}\right)$. Note that $f$ is the Pfaffian of $\left(\left\{f_{i}, f_{j}\right\}_{0}\right)$. Consider the $(2 n-2)$-form

$$
\Phi=-\frac{1}{f}\left(\sigma+\frac{g}{k-1} \omega_{0}\right) \wedge \frac{\omega_{0}^{k-2}}{(k-2) !} \wedge d f_{1} \wedge \ldots \wedge d f_{2 n-2 k},
$$

where $\sigma$ is a 2 -form on $M$ satisfying:

$$
2 \sigma \wedge \delta(\sigma)=\delta(\sigma \wedge \sigma)
$$

The operator $\delta$ is defined by $\delta=* d *$, where $*$ is the standard star operator.

(ii) The 2-form $\sigma$ is a section of $\bigwedge^{2} D^{\circ}$ of maximal rank where $D^{\circ}$ is the annihilator of the distribution $D$ generated by the vector fields of $X_{f_{1}}, \ldots, X_{f_{2 n-2 k}}$.

Finally, $g=i_{\Lambda_{0}} \sigma$. Then $\Phi$ corresponds to a Poisson tensor field $\Lambda$ on $M$ with orbits of dimension at most $2 k$ for which $f_{1}, \ldots, f_{2 n-2 k}$ are Casimir functions. Precisely, $\Lambda=\Lambda_{0}^{\#}(\sigma)$ and the associated bracket of $\Lambda$ on $C^{\infty}(M)$ is given, for any $h_{1}, h_{2} \in$ $C^{\infty}(M)$, by

$$
\begin{aligned}
\left\{h_{1}, h_{2}\right\} \Omega= & -\frac{1}{f} d h_{1} \wedge d h_{2} \wedge\left(\sigma+\frac{g}{k-1} \omega_{0}\right) \wedge \frac{\omega_{0}^{k-2}}{(k-2) !} \\
& \wedge d f_{1} \wedge \ldots \wedge d f_{2 n-2 k}
\end{aligned}
$$

Conversely, if $\Lambda$ is a Poisson tensor on $\left(M, \omega_{0}\right)$ of rank at most $2 k$ on an open and dense subset $\mathcal{U}$ of $M$, then there are $2 n-2 k$ functionally independent smooth functions $f_{1}, \ldots, f_{2 n-2 k}$ on $\mathcal{U}$ and a suitable 2 -form $\sigma$ on $M$ such that $\Psi_{\Lambda}=-i_{\Lambda} \Omega$ and $\{\cdot, \cdot\}$ is of the form (19).
Similar results hold when $M$ is an odd-dimensional manifold. One may establish a similar formula for the Poisson brackets on $C^{\infty}(M)$ with the prescribed properties. For this construction, we assume that $M$ is equipped with a suitable almost cosymplectic structure $\left(\vartheta_{0}, \Theta_{0}\right)$ and with the volume form $\Omega=\vartheta_{0} \wedge \frac{\Theta_{0}^{n}}{n !}$. In Damianou and Petalidou [29] we showed how one obtains the $A_{n}$ Volterra bracket starting from the $A_{n}$ Lie Poisson bracket of the periodic Toda lattice. The algorithm can of course be generalized to any complex simple Lie algebra.

\section{FROM $A_{N}$-PERIODIC TODA TO VOLTERRA LATTICE}

In this section we describe the $A_{n}$-Toda to $A_{n}$-Volterra case. We begin with the linear Poisson structure $\Lambda_{T}$ associated with the periodic Toda lattice of $n$ particles. This Poisson structure has two well-known Casimir functions. Using formula (19) we construct another Poisson structure having the same Casimir invariants with $\Lambda_{T}$. It turns out that this structure decomposes as a direct sum of two Poisson tensors one of which (involving only the $a$ variables in Flaschka's coordinates) is the quadratic Poisson bracket of the Volterra lattice.

The periodic Toda lattice of $n$ particles $(n \geq 2)$ is the system of ordinary differential equations on $\mathbb{R}^{2 n}$ which in Flaschka's [7] coordinates $\left(a_{1}, \ldots, a_{n}, b_{1}, \ldots, b_{n}\right)$ takes the form

$$
\begin{gathered}
\dot{a}_{i}=a_{i}\left(b_{i+1}-b_{i}\right) \quad \text { and } \quad \dot{b}_{i}=2\left(a_{i}^{2}-a_{i-1}^{2}\right) \\
\left(i \in \mathbb{Z} \text { and }\left(a_{i+n}, b_{i+n}\right)=\left(a_{i}, b_{i}\right)\right) .
\end{gathered}
$$

This system is Hamiltonian with respect to the nonstandard LiePoisson structure

$$
\Lambda_{T}=\sum_{i=1}^{n} a_{i} \frac{\partial}{\partial a_{i}} \wedge\left(\frac{\partial}{\partial b_{i}}-\frac{\partial}{\partial b_{i+1}}\right)
$$

on $\mathbb{R}^{2 n}$ and it has as Hamiltonian the function $H=\sum_{i=1}^{n}\left(a_{i}^{2}+\right.$ $\left.\frac{1}{2} b_{i}^{2}\right) . \Lambda_{T}$ is of rank $2 n-2$ on $\mathcal{U}=\left\{\left(a_{1}, \ldots, a_{n}, b_{1}, \ldots, b_{n}\right) \in\right.$ $\left.\mathbb{R}^{2 n} / \sum_{i=1}^{n} a_{1} \ldots a_{i-1} a_{i+1} \ldots a_{n} \neq 0\right\}$ and it admits two Casimir functions:

$$
C_{1}=b_{1}+b_{2}+\ldots+b_{n} \text { and } C_{2}=a_{1} a_{2} \ldots a_{n} .
$$

We consider on $\mathbb{R}^{2 n}$ the standard symplectic form $\omega_{0}=\sum_{i=1}^{n} d a_{i} \wedge d b_{i}$, its associated Poisson tensor $\Lambda_{0}=\sum_{i=1}^{n} \frac{\partial}{\partial a_{i}} \wedge \frac{\partial}{\partial b_{i}}$, and the corresponding volume element $\Omega=\frac{\omega_{0}^{n}}{n !}=d a_{1} \wedge d b_{1} \wedge \ldots \wedge d a_{n} \wedge d b_{n}$. The Hamiltonian vector fields of $C_{1}$ and $C_{2}$ with respect to $\Lambda_{0}$ are

$X_{C_{1}}=-\sum_{i=1}^{n} \frac{\partial}{\partial a_{i}} \quad$ and $\quad X_{C_{2}}=\sum_{i=1}^{n} a_{1} \ldots a_{i-1} a_{i+1} \ldots a_{n} \frac{\partial}{\partial b_{i}}$. 
So, $D=\left\langle X_{C_{1}}, X_{C_{2}}\right\rangle$ and

$$
\begin{aligned}
D^{\circ}= & \left\{\sum_{i=1}^{n}\left(\alpha_{i} d a_{i}+\beta_{i} d b_{i}\right) \in \Omega^{1}\left(\mathbb{R}^{2 n}\right) / \sum_{i=1}^{n} \alpha_{i}=0\right. \text { and } \\
& \left.\sum_{i=1}^{n} a_{1} \ldots a_{i-1} \beta_{i} a_{i+1} \ldots a_{n}=0\right\} .
\end{aligned}
$$

The family of 1 -forms $\left(\sigma_{1}, \ldots, \sigma_{n-1}, \sigma_{1}^{\prime}, \ldots, \sigma_{n-1}^{\prime}\right)$,

$$
\begin{aligned}
\sigma_{j} & =d a_{j}-d a_{j+1} \text { and } \sigma_{j}^{\prime}=a_{j} d b_{j}-a_{j+1} d b_{j+1}, \\
j & =1, \ldots, n-1,
\end{aligned}
$$

provides, at every point $(a, b) \in \mathcal{U}$, a basis of $D_{(a, b)}^{\circ}$. The section of maximal rank $\sigma_{T}$ of $\Lambda^{2} D^{\circ} \rightarrow \mathcal{U}$, which corresponds to $\Lambda_{T}$, via the isomorphism $\Lambda_{0}^{\#}$, and verifies (18), is written, in this basis, as

$$
\sigma_{T}=\sum_{j=1}^{n-1} \sigma_{j} \wedge\left(\sum_{l=j}^{n-1} \sigma_{l}^{\prime}\right)
$$

Now, we consider on $\mathbb{R}^{2 n}$ the 2 -form

$$
\begin{aligned}
\sigma= & \sum_{j=1}^{n-2} \sigma_{j} \wedge\left(\sum_{l=j+1}^{n-1} \sigma_{l}\right)+\sum_{j=1}^{n-2} \sigma_{j}^{\prime} \wedge\left(\sum_{l=j+1}^{n-1} \sigma_{l}^{\prime}\right) \\
= & \sum_{j=1}^{n-2}\left[\left(d a_{j}-d a_{j+1}\right) \wedge\left(d a_{j+1}-d a_{n}\right)\right. \\
& \left.\quad+\left(a_{j} d b_{j}-a_{j+1} d b_{j+1}\right) \wedge\left(a_{j+1} d b_{j+1}-a_{n} d b_{n}\right)\right] \\
= & \sum_{j=1}^{n}\left(d a_{j} \wedge d a_{j+1}+a_{j} a_{j+1} d b_{j} \wedge d b_{j+1}\right) .
\end{aligned}
$$

It is a section of $\bigwedge^{2} D^{\circ}$ whose rank depends on the parity of $n$; if $n$ is odd, its rank is $2 n-2$ on $\mathcal{U}$, while, if $n$ is even, its rank is $2 n-4$ almost everywhere on $\mathbb{R}^{2 n}$. Also, after a long computation, we can confirm that it satisfies (18). Thus, its image via $\Lambda_{0}^{\#}$, i.e., the bivector field

$$
\Lambda=\sum_{j=1}^{n}\left(a_{j} a_{j+1} \frac{\partial}{\partial a_{j}} \wedge \frac{\partial}{\partial a_{j+1}}+\frac{\partial}{\partial b_{j}} \wedge \frac{\partial}{\partial b_{j+1}}\right)
$$

defines a Poisson structure on $\mathbb{R}^{2 n}$ with symplectic leaves of dimension at most $2 n-2$, when $n$ is odd, that has $C_{1}$ and $C_{2}$ as Casimir functions. (When $n$ is even, $\Lambda$ has two more Casimir functions.) We remark that $\left(\mathbb{R}^{2 n}, \Lambda\right)$ can be viewed as the product of Poisson manifolds $\left(\mathbb{R}^{n}, \Lambda_{V}\right) \times\left(\mathbb{R}^{n}, \Lambda^{\prime}\right)$, where

$$
\begin{aligned}
\Lambda_{V} & =\sum_{j=1}^{n} a_{j} a_{j+1} \frac{\partial}{\partial a_{j}} \wedge \frac{\partial}{\partial a_{j+1}} \quad \text { and } \\
\Lambda^{\prime} & =\sum_{j=1}^{n} \frac{\partial}{\partial b_{j}} \wedge \frac{\partial}{\partial b_{j+1}} .
\end{aligned}
$$

The Poisson tensor $\Lambda_{V}$ is the quadratic Poisson structure associated to the periodic Volterra lattice

$$
\dot{a}_{i}=a_{i}\left(a_{i+1}-a_{i-1}\right), \quad i=1, \ldots, n, \text { with } a_{n+i}=a_{i},
$$

on $\mathbb{R}^{n}$ and it has $C_{2}$ as unique Casimir function, when $n=2 k+1$ is odd.

It is well known that (21) is a completely integrable system that admits a bihamiltonian formulation, [30-32], and a Lax pair representation $[8,9,33] . \Lambda_{V}$ is compatible with the cubic Poisson tensor field $Q$ on $\mathbb{R}^{n}$ whose components are the functions

$$
\begin{aligned}
Q_{i j}= & a_{i} a_{j}\left(a_{i}+a_{j}\right)\left(\delta_{i+1, j}-\delta_{j+1, i}\right)+a_{i} a_{i+1} a_{i+2} \delta_{i+2, j} \\
& -a_{i} a_{i-1} a_{i-2} \delta_{i-2, j},
\end{aligned}
$$

and we have that

$$
\Lambda_{V}^{\#}(d H)=Q^{\#}\left(d \ln C_{2}\right)
$$

Also, (21) can be written in the form $\dot{L}=[B, L]$, where

$$
L=\left(\begin{array}{cccccc}
0 & \sqrt{a_{1}} & 0 & \ldots & 0 & \sqrt{a_{n}} \\
\sqrt{a_{1}} & 0 & \sqrt{a_{2}} & \ddots & & 0 \\
0 & \ddots & \sqrt{a_{2}} & \ddots & \ddots & \vdots \\
\vdots & \ddots & \ddots & \ddots & \ddots & 0 \\
0 & & \ddots & \ddots & 0 & \sqrt{a_{n-1}} \\
\sqrt{a_{n}} & 0 & \ldots & 0 & \sqrt{a_{n-1}} & 0
\end{array}\right)
$$

and

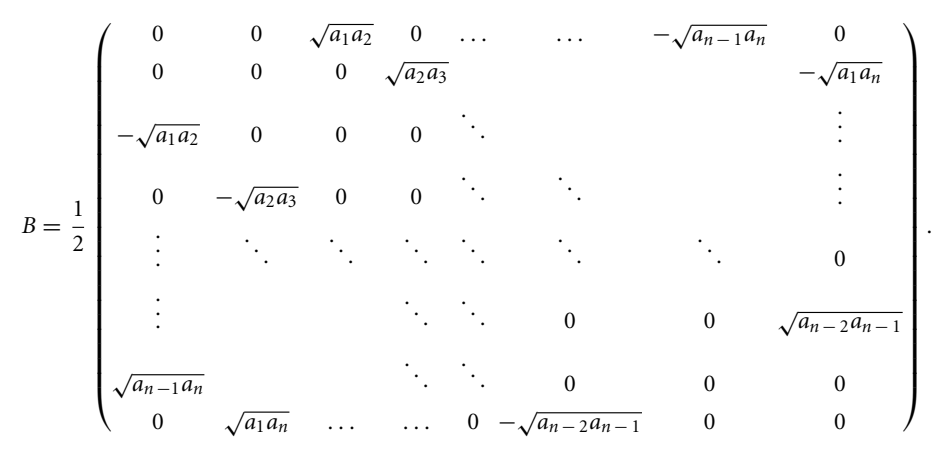

The functions det $L=2 C_{2}^{1 / 2}$ and $\operatorname{Tr} L^{2 k}$ are first integrals of (21). 
Some other $A_{n}$-type Lotka-Volterra systems: We remark that to each 2 -form $\sigma^{\prime}$ which is a linear combination of 2forms of type $\sigma_{i}^{\prime} \wedge \sigma_{j}^{\prime}$ corresponds, via $\Lambda_{0}^{\#}$, a diagonal quadratic Poisson structure whose a Casimir is the function $C_{2}$ and whose Hamiltonian system associated to $H=a_{1}+\ldots+a_{n}$ is of the form (4). For example, if $n=5$ and $\sigma^{\prime}=3 \sigma_{1}^{\prime} \wedge \sigma_{2}^{\prime}+\sigma_{1}^{\prime} \wedge \sigma_{3}^{\prime}+$ $\sigma_{1}^{\prime} \wedge \sigma_{4}^{\prime}-2 \sigma_{2}^{\prime} \wedge \sigma_{4}^{\prime}+\sigma_{3}^{\prime} \wedge \sigma_{4}^{\prime}$,

$$
\Lambda=\Lambda_{0}^{\#}\left(\sigma^{\prime}\right)=\left(\begin{array}{ccccc}
0 & 3 a_{1} a_{2} & -2 a_{1} a_{3} & 0 & -a_{1} a_{5} \\
-3 a_{1} a_{2} & 0 & 2 a_{2} a_{3} & -2 a_{2} a_{4} & 3 a_{2} a_{5} \\
2 a_{1} a_{3} & -2 a_{2} a_{3} & 0 & 3 a_{3} a_{4} & -3 a_{3} a_{5} \\
0 & 2 a_{2} a_{4} & -3 a_{3} a_{4} & 0 & a_{4} a_{5} \\
a_{1} a_{5} & -3 a_{2} a_{5} & 3 a_{3} a_{5} & -a_{4} a_{5} & 0
\end{array}\right)
$$

and the Hamiltonian vector field $\Lambda^{\#}(d H)$ corresponds to the system

$$
\begin{aligned}
& \dot{a}_{1}=a_{1}\left(3 a_{2}-2 a_{3}-a_{5}\right) \\
& \dot{a}_{2}=a_{2}\left(-3 a_{1}+2 a_{3}-2 a_{4}+3 a_{5}\right) \\
& \dot{a}_{3}=a_{3}\left(2 a_{1}-2 a_{2}+3 a_{4}-3 a_{5}\right) \\
& \dot{a}_{4}=a_{4}\left(2 a_{2}-3 a_{3}+a_{5}\right) \\
& \dot{a}_{5}=a_{5}\left(a_{1}-3 a_{2}+3 a_{3}-a_{4}\right) .
\end{aligned}
$$

The integrability of the Lotka-Volterra systems obtained by the above procedure is an open problem.

We close with the following observation. Beginning with the standard Poisson bracket for the periodic Toda lattice corresponding to a complex simple Lie algebra $\mathfrak{g}$ and by repeating the procedure of this section we produce new Lotka-Volterra systems associated with $\mathfrak{g}$. Establishing the integrability of these systems is also an open problem.

\section{REFERENCES}

1. Lotka AJ. Undamped oscillations derived from the law of mass action. J Am Chem Soc. (1920) 42, 1595-9. doi: 10.1021/ja01453a010

2. Hernandez-Bermejo B, Fairen V. Separation of variables in the Jacobi identities, Phys Lett A (2000) 271, 258-63. doi: 10.1016/S0375-9601(00) 00375-3

3. Volterra V. Leçons sur la théorie mathématique de la lutte pour la vie, (1931) Paris: Gauthier-Villars.

4. Plank M. Hamiltonian structures for the $n$-dimensional Lotka-Volterra equations. J Math Phys. (1995) 36, 3520-34. doi: 10.1063/1.530978

5. Bogoyavlensky OI. Integrable Lotka-Volterra systems. Regul Chaotic Dyn. (2008) 13, 543-56. doi: 10.1134/S1560354708060051

6. Kac M, van Moerbeke P. On an explicit soluble system of nonlinear differential equations related to certain Toda lattices. Adv Math. (1975) 16, 160-9. doi: 10.1016/0001-8708(75)90148-6

7. Flaschka H. On the Toda lattice II. Inverse scattering solution. Progr Theor Phys. (1974) 51, 703-16. doi: 10.1143/PTP.51.703

8. Moser J. Three integrable Hamiltonian systems connected with isospectral deformations. Adv Math. (1975) 16, 197-220. doi: 10.1016/00018708(75)90151-6

9. Damianou PA. The Volterra model and its relation to the Toda lattice. Phys Lett A (1991) 155, 126-32. doi: 10.1016/0375-9601(91)90578-V

10. Antoniou P, Pitsillides A. "Congestion control in autonomous decentralized networks based on the Lotka-Volterra competition model," In: ICAAN 2009 19th International Conference on Artificial Neural Networks, (Limassol, Cyprus), pp. 14-7, (2009).

11. Ballesteros A, Blasco A Musso F. Integrable deformations of LotkaVolterra systems Phys Lett A (2011) 375, 3370-4. doi: 10.1016/j.physleta.2011.07.055
12. Goriely A. Integrability and Nonintegrability of Dynamical Systems, Singapore: World Scientific Publishing, (2001).

13. Hernandez-Bermejo B, Fairen, V. Hamiltonian structure and Darboux theorem for families of generalized Lotka-Volterra systems. J Math Phys. (1998) 39, 6162-74. doi: 10.1063/1.532621

14. Plank M. Bi-Hamiltonian systems and Lotka-Volterra equations: a threedimensional classification. Nonlinearity (1996) 9, 887-96. doi: 10.1088/0951$7715 / 9 / 4 / 004$

15. Cairó L, Feix MR. Families of invariants of the motion for the Lotka-Volterra equations: the linear polynomial family. J Math Phys. (1992) 33, 2440-55. doi: $10.1063 / 1.529614$

16. Cairó L, Llibre J. Darboux integrability for 3D Lotka-Volterra systems. J Phys A Math Gen. (2000) 33, 2395-406. doi: 10.1088/0305-4470/33/12/307

17. Labrunie $S$. On the polynomial first integrals of the (a, b, c) Lotka-Volterra system. J Math Phys. (1996) 37, 5539-50. doi: 10.1063/1.531721

18. Maciejewski AJ, Przybylska M. Darboux polynomials and first integrals of natural polynomial Hamiltonian systems. Phys Lett A (2004) 326, 219-26. doi: 10.1016/j.physleta.2004.04.034

19. Moulin-Ollagnier J. Polynomial first integrals of the Lotka-Volterra system. Bull Sci Math. (1997) 121, 463-76.

20. Moulin-Ollagnier J. Rational integration of the Lotka-Volterra system. Bull Sci Math. (1999) 123, 437-66. doi: 10.1016/S0007-4497(99)00111-6

21. Fernandes RL, Oliva WM. "Hamiltonian dynamics of the Lotka-Volterra equations," In: International Conference on Differential Equations, Lisboa 1995 (River Edge, NJ: World Sci. Publ.) (1998) pp. 327-34.

22. Bogoyavlensky OI. Five constructions of integrable Hamiltonian systems. Acta Appl Math. (1988) 13, 227-66.

23. Bogoyavlensky OI. Intergrable discretizations of the KdV equation. Phys Lett A (1988) 134, 34-8. doi: 10.1016/0375-9601(88)90542-7

24. Kouzaris SP. Multiple Hamiltonian structures and Lax pairs for Bogoyavlensky-Volterra systems. J Nonlinear Math Phys. (2003) 10, 431-50. doi: 10.2991/jnmp.2003.10.4.2

25. Damianou PA, Fernandes RL. From the Toda lattice to the Volterra lattice and back. Rep Math Phys. (2002) 50, 361-78. doi: 10.1016/S0034-4877(02)80066-0

26. Damianou PA. "Lotka-Volterra systems associated with graphs," In: Sixth Workshop Group Analysis of Differential Equations and Integrable Systems (2013), 30-44

27. Charalambides SA, Damianou PA, Evripidou CA. On generalized Volterra systems. arXiv:1305.7329, (2013).

28. Evripidou CA. Applications of Root Systems in Geometry and Physics. Ph.D. dissertation, University of Cyprus (2014)

29. Damianou PA, Petalidou F. Poisson brackets with prescribed Casimirs. Can Math. (2012) 64, 991-1018. doi: 10.4153/CJM-2011-082-2

30. Penskoï AV. Canonically conjugate variables for the Volterra system with periodic boundary conditions. Mat Zametki. (1998) 68 115-28. (English translation: Math. Notes. (1998) 64 98-109.) doi: 10.1007/BF02307200

31. Veselov AP, Penskoï AV. On algebro-geometric Poisson brackets for the Volterra lattice. Reg Chaot Dyn. (1998), 3, 3-9. doi: 10.1070/rd1998v003n 02ABEH000066

32. Falqui G, Pedroni M. Gel'fand-Zakharevich systems and algebraic integrability: The Volterra lattice revised. Reg Chaot Dyn. (2005) 10, 399-412. doi: 10.1070/RD2005v010n04ABEH000322

33. Fernandes RL, Santos JP. Integrability of the Periodic KM System. Rep Math Phys. (1997) 40 475-84. doi: 10.1016/S0034-4877(97)85896-X

Conflict of Interest Statement: The authors declare that the research was conducted in the absence of any commercial or financial relationships that could be construed as a potential conflict of interest.

Received: 01 June 2014; accepted: 28 July 2014; published online: 19 August 2014. Citation: Damianou PA and Petalidou F (2014) On the liouville intergrability of Lotka-Volterra systems. Front. Phys. 2:50. doi: 10.3389/fphy.2014.00050

This article was submitted to Mathematical Physics, a section of the journal Frontiers in Physics.

Copyright (c) 2014 Damianou and Petalidou. This is an open-access article distributed under the terms of the Creative Commons Attribution License (CC BY). The use, distribution or reproduction in other forums is permitted, provided the original author(s) or licensor are credited and that the original publication in this journal is cited, in accordance with accepted academic practice. No use, distribution or reproduction is permitted which does not comply with these terms. 